factors such as anxiety and depression (HADS), sleep quality (PSQI) and physical exercise (GPAQ).

Results 759/1919 IBD patients in clinical remission (39.6\%) reported fatigue in the past 2 weeks, while 1034 patients (53.9\%) did not report fatigue. Patients who reported fatigue were more frequently female, had more frequently $C D$, and were more frequently smokers (Table 1). Univariable comparisons showed higher inflammatory markers in the fatigued group, with fewer patients in clinical remission. Multivariable analyses identified female sex (OR 2.4), CRP $>5$ (OR 2.1), bad sleep quality (OR 2.5), anxiety (OR 1.8) and depression (OR 6.2) as independent factors associated with fatigue.

Conclusion We show the significant burden of fatigue in IBD patients and describe putative causes which demonstrate both the impact of residual gut inflammation and the relationship between fatigue and mental health.

\section{PMO-51 IS CLIPPER A SUITABLE TREATMENT OPTION FOR COLITIS PATIENTS?}

Chandni Radia*, Akudo Nwaogu, Efsaia Savvoulidou, Bu'Hussain Hayee, Shadwa AbdelAziz, Patrick Dubois, Alexandra Kent. King's College Hospital, London, UK

\subsection{6/gutjpl-2021-BSG.190}

Introduction The gut selective oral beclomethasone dipropionate (Clipper) is approved for use in mild-to-moderate ulcerative colitis (UC), with a better safety profile compared to conventional systemic corticosteroids. We reviewed the efficacy of Clipper in inducing clinical remission in patients at our Trust.

Methods We collated data on patients identified though the Pharmacy database as receiving a Clipper prescription from February 2018 to February 2021. Disease activity was determined using the Simple Clinical Colitis Activity Index (SCCAI) at baseline, 4 weeks and 6 months to assess clinical remission and response rates. Where available, faecal calprotectin levels were collected alongside information on background medication changes.

Results A total of 235 patients with UC were prescribed Clipper in the given period. 39 were excluded from analysis. 196 patients had 4 week outcome data and 176 also had 6 month data; median age 39 years (range 17-94 years). There were no significant side effects.

The average SCCAI at baseline was $6.3 .31 \%$ of patients had their background medication escalated alongside Clipper treatment. Clinical response and remission rates at 4 weeks were $71 \%$ and $53 \%$ respectively. Remission rates were significantly higher in those with mild activity (SCCAI $\leq 6,65 \%$ ) compared to those with moderate or severe activity (SCCAI $>6,31 \%)(p=0.00001)$. At 6 months, $42 \%$ of patients remained in steroid-free clinical remission, $43 \%$ of whom had their background therapy escalated either alongside Clipper or during the 6 month follow up. 57\% of those in remission at 4 weeks remained in steroid-free clinical remission at 6 months. 69 patients had paired faecal calprotectin levels at time of Clipper prescription (mean $854 \mathrm{ug} / \mathrm{g}$ ) and at 6 months (mean 299ug/g), showing a statistically significant reduction $(\mathrm{p}=0.0009) .17$ patients $(8.7 \%)$ required admission for ongoing active disease, and 30\% required at least one further course of steroids.
30 patients with colonic Crohn's Disease (CD) were also prescribed Clipper. Clinical remission and response rates were $60 \%$ and $70 \%$ respectively at 4 weeks. $83 \%$ of those in remission remained in steroid-free clinical remission at 6 months. Overall 53\% were in remission at 6 months, 4 of whom had their background medications escalated over the review period. 12 patients had paired faecal calprotectin results showing a mean reduction of $981 \mathrm{ug} / \mathrm{g}(\mathrm{p}=0.036)$.

Conclusions Clinical remission can be effectively induced with Clipper in patients with UC, with significantly higher remission in mild disease. It is useful as bridging therapy during a change or escalation in background therapy. It has the potential to reduce the need for systemic steroid therapy. Clipper seems to also have a similar effect in patients with colonic CD.

\section{Liver}

\section{PWE-1 ALKBH5-MODIFIED HMGB1-STING ACTIVATION CONTRIBUTES TO RADIATION INDUCED LIVER DISEASE VIA INNATE IMMUNE RESPONSE}

Genwen Chen*, Qianqian Zhao, Baoying Yuan, Zhaochong Zeng. Zhongshan Hospital, Fudan University, Shanghai, China

\subsection{6/gutjnl-2021-BSG.191}

Introduction Radiation therapy (RT) is vital for the therapy of primary liver cancer, but inevitable liver injury limits the implement of RT. N6-methyladenosine (m6A) methylation is involved in many molecular functions; however, its role in radiation-induced liver diseases (RILD) remains unknown. Hence, we intend to investigate the role of m6A methylation in RILD.

Methods Methylated RNA-immunoprecipitation sequencing (MeRIP-seq) and RNA transcriptome sequencing (RNA-seq) were used to reveal the methylation pattern of human hepatic stellate cells with exposure to irradiation. $\mathrm{C} 3 \mathrm{H} / \mathrm{HeN}$ mice and STING-deficient mice underwent X-ray irradiation of $24 \mathrm{~Gy}$ in three fractions. The m6A methylation of HMGB1 transcript was validated using MeRIP, RIP, luciferase assay and mRNA decay assays.

Results Human hepatic stellate cells shown significant difference of methylation pattern after 8 Gy of X-ray irradiation. Irradiation recruits ALKBH5, an eraser of m6A methylation, and then demethylated HMGB1 transcript at m6A residues in the 3'UTR, following activation of STING-IRF3 signaling. Inserting of the HMGB1 3'UTR into a luciferase reporter resulted in regulation of luciferase activity by ALKBH5 knockdown, which was lost after m6A residue mutation. Strikingly, ALKBH5 deficiency or HMGB1 silencing both attenuated type I interferon production, resulting to less hepatocyte apoptosis. In vivo depletion of ALKBH5 abolished the upregulation of HMGB1-mediated STING signaling, leading to slightly liver inflammation, which was consistent to $\mathrm{STING}^{-/-}$mice in response to irradiation. Notably, the $\mathrm{m} 6 \mathrm{~A}$ reader protein YTHDF2 directly binds to m6A-modified site of HMGB1 transcript, which consequently promotes its degradation.

Conclusions ALKBH5-dependent HMGB1 expression mediates STING-IRF3 innate immune response in RILD. 